\title{
Les écoulements diphasiques d'un mélange de gaz et de liquide dans les conduites de grande longueur. Problèmes spécifiques de métrologie
}

\author{
Two-phase flow of a gas/liquid mixture in a long pipe, \\ specific metrology problems
}

\author{
Jean-Michel Fitremann* \\ Agrégé de Sciences Physiques \\ Docteur ès-Sciences
}

\begin{abstract}
L'extraction d'huile brute des gisements souterrains et son transport provoque l'apparition de gaz par détente et établit ainsi un écoulement diphasique dans les puits et les réseaux de collecte. Il en est de même des gisements de gaz où la détente provoque l'apparition de liquide par condensation rétrograde. Les systèmes de mesure et de traitement des signaux dans les expériences de simulation imaginées par les chercheurs du groupe DIP sont adaptés aux écoulements le plus fréquemment rencontrés dans ces applications, à savoir :
\end{abstract}

- les écoulements par bouchons ascendants verticaux (Fig. 1.a)

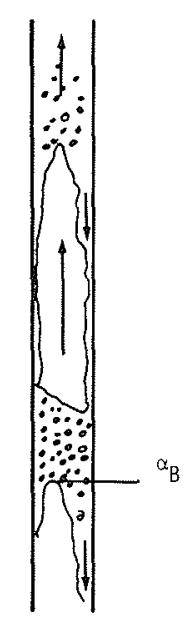

Figure 1.a -... Ecoulement à poches vertical à nombre de Bond élevé

(*) Groupe de Recherches sur la Dynamique des Ecoulements Diphasiques (DIP) - Bâtiment $502-91405$ Orsay.

(1) $\rho_{\mathrm{L}}$ : masse volumique du liquide

$\mathrm{g}$ : accélération de la pesanteur

$\sigma$ : tension in terfaciale

D : diamètre de la conduite
- les écoulements par bouchons en conduite horizontale ou faiblement inclinée (Fig. 1.b)

- les écoulements stratifiés turbulents (Fig. 1.c)

Dans les deux premiers cas, le nombre de Bond $\underline{\rho_{\mathrm{L}} \mathrm{gD}^{2}}(1)$

$\sigma$

quide soient en réalité formés d'un lit dense de bulles de petit diamètre.

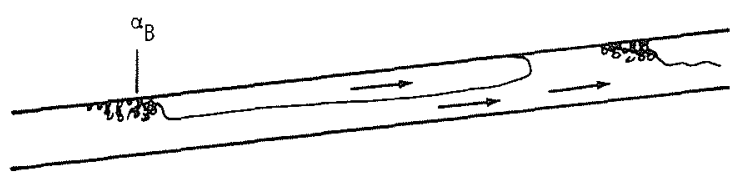

Figure 1.b - Ecoulement à poches oblique

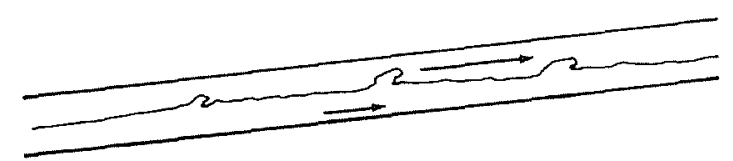

Figure 1.c - Ecoulement stratifié à vagues

Ecoulements par bouchons ascendants

Le modèle mathématique issu de l'application des théorèmes généraux des écoulements á deux phases $[1,2]$ montre qu'il est nécessaire de mesurer au moins le taux de présence relatif de bulles dans le bouchon $\alpha_{B}$ et sa répartition dans une section, la fonction de présence de phase dans une poche de gaz et sa répartition dans une section, la vitesse de déplacement des poches de gaz, la contrainte de frottement à la paroi et sa variation dans le temps et avec la position sur le périmètre, enfin le gradient de pression total. 
Les deux premières grandeurs sont obtenues par sonde optique de présence de phase AID [3]. Un tri en temps réel ou différé [4] [5] des intervalles de temps de présence du gaz longs ou courts permet de séparer la contribution des bulles et des poches au transport du gaz et l'intégration des profils [1] fournit les moyennes désirées. A l'aide de deux sondes éloignées, on peut par une méthode de reconnaissance de signaux obtenir la densité de probabilité des vitesses des bulles ou des poches de gaz [2].

Le calcul de la densité moyenne, l'évaluation des termes d'accélération dans le bilan d'impulsion, permet de calculer la contribution du frottement au gradient de pression. Dans une large plage de régimes en écoulement vertical, celui-ci est dirigé vers le bas en moyenne, $[1,6]$, le frottement dans les films descendant autour des poches de gaz l'emportant sur celui des bouchons de liquide dirigé, lui, vers le haut comme dans un écoulement habituel. L'analyse du frottement pariétal instantanée revêt donc une grande importance pour l'étude de cette configuration très complexe. La méthode polarographique, décrite par ailleurs par G. Cognet, a été choisie comme la plus convenable pour ce genre d'etude. Dans le cas d'un écoulement oblique ou horizontal, la mesure sur tout un périmètre de conduite s'impose, naturellement. Disons enfin, que la mesure des gradients de pression nécessite des précautions particulières [1].

\section{Ecoulements stratifiés turbulents}

La figure 2 a montre une coupe d'un tel écoulement. Rien ne garantit que la surface moyenne de l'interface soit plane. C'est pourtant ce que l'on observe lorsque les vitesses ne sont pas trop grandes et le modèle mathématique en est d'autant simplifié. Les lois physiques nécessaires pour le calcul concernent les valeurs moyennes du frottement sur le périmètre gaz $P_{G}$, le périmètre liquide $P_{L}$ et l'interface $P_{j}$. La mesure de ces troisquantités n'est pas simple, en particulier, la mesure du frot-

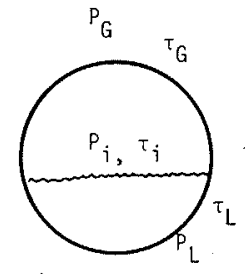

a)

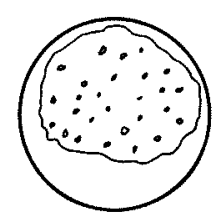

b)
Figure 2 - Coupe d'un écoulement stratifié, a) à vitese réduite, b) à vitesse élevée. tement interfacial $\tau_{i}$. Nous avons choisi une méthode de bilan sur un tronçon de conduite faiblement inclinable où la mesure très précise du gradient de pression de la pente et des débits permet le calcul. Cette expérience très délicate donne des résultats qui doivent être confrontés à d'autres évaluations basées sur la mesure de profils de vitesses et de contraintes de Reynolds [7].

Aux fortes vitesses, l'interface n'est plus plane et l'on observe un entrainement de gouttelettes fines dans la phase gazeuse. Aucune technique métrologique ne semble vraiment adaptée à l'étude détaillée de tels écoulements (Fig. 2.b) : films liquides minces fortement ondulés, écoulements à brouillard, etc...

\section{Conclusions}

L'industrie pétrolière, dont les besoins en modèles de prédiction des propriétés des écoulements diphasiques sont importants, fait largement appel aux techniques de mesures diphasiques décrites ailleurs dans cette revue avec des méthodes de traitement originales. Des techniques spéciales sont parfois nécessaires, comme pour la mesure du frottement par exemple, dans l'étude de configurations qui ne sont abondantes que dans ce secteur de l'industrie. Pour l'avenir, il est souhaitable de voir se développer des méthodes adaptées aux configurations à film mince et à brouillards (forts taux de gaz).

\section{Bibliographie}

[1] FITREMANN J.-M. - Théorie des écoulements diphasiques gaz-liquide et applications à l'étude de quelques régimes d'écoulements verticaux ascendants. Thèse de doctorat, 1977, Paris.

[2] FERRE D. - Etude expérimentale des écoulements diphasiques a poches en conduites horizontales. Thèse de $3^{\circ}$ cycle, 1978, Paris.

[3] GALAUP J.P. - Contribution à l'étude des méthodes de mesure on écoulement diphasique. Thèse de Docteur-Ingénieur, 1975, Grenoble.

[4] FITREMANN J.-M., POSTAIRE J.G., GULLPIN Ch. Méthode de différenciation automatique des bulles et des poches dans les écoulements à deux phases gaz-liquide et de mesure de leurs vitesses respectives. Symposium AIRHSHF, Grenoble 1976.

[5] POSTAIRE J.G. et FITREMANN J.M. - A Digital Special Purpose Signal Processor for Two-Phase Flow Real-Time Analysis. IEEE Trans. Inst. Meas., Vol IM $26 \mathrm{~N}^{\circ}$ 3, Sept. 77

[6] TRIKI T. - Thèse de Docteur-Ingénieur, Paris, 1978.

[7] SUZANNE Ch. - Etude expérimentale de l'écoulement turbulent stratifié d'un gaz et d'un liquide. - Thèse de Docteur-Ingénieur, Université Paul Sabatier, Toulouse, 1977. 